䗆 


\section{Progress in Physics}

Volume 18

Series Editors

Anne Boutet de Monvel

Gerald Kaiser

Picture on the cover is a simplified version of a figure created by Oliver Conradt (Department of Physics and Astronomy, University of Basel, Klingelbergstrasse 82, CH-4056 Basel, Switzerland). It depicts projective plane coordinates of a pencil of planes. For more information we refer to Conradt's paper The Principle of Duality in Clifford Algebra and Projective Geometry in this volume. 
Clifford Algebras and their Applications in Mathematical Physics

Volume 1: Algebra and Physics

Rafał Ablamowicz

Bertfried Fauser

Editors

Springer Science+Business Media, LLC 


\author{
Rafal Ablamowicz \\ Department of Mathematics \\ Box 5054 \\ Tennessee Technological University \\ Cookeville, TN 38505 \\ U.S.A.
}

\author{
Bertfried Fauser \\ Fachbereich Physik \\ Fach M678 \\ Universität Konstanz \\ 78457 Konstanz \\ Germany
}

\section{Library of Congress Cataloging-in-Publication Data}

Clifford algebras and their applications in mathematical physics.

p. cm. - (Progress in physics ; v. 18-19)

Includes bibliographical references and indexes.

Contents: v. 1. Algebra and physics / Rafał Ablamowicz, Bertfried Fauser, editors-v.

2. Clifford analysis / John Ryan, Wolfgang Sprößig, editors.

ISBN 978-1-4612-7116-1 ISBN 978-1-4612-1368-0 (eBook)

DOI 10.1007/978-1-4612-1368-0

1. Clifford algebras. 2. Mathematical physics. I. Ablamowicz, Rafał. II. Progress in physics (Boston, Mass.); v. 18-19.

QC20.7.C55 C55 2000

$530.15 ' 257-\mathrm{dc} 21$

00-034310

AMS Subject Classifications: 03G12, 03G25, 08A99, 14A22, 14L35, 14N10, 15A03, 15A18, 15A23, 15A33, 15A63, 15A66, 16W30, 16W50, 17A35, 17B10, 17B37, 17C90, 30C30, 22E60, 32L 25, 34A26, 51A25, 51B10, 51B20, 51P05, 53A50, 53B25, 53B 30, 53B99, 53C20, 53C50, 53Z05, 54H15, 58A99, 68T15, 70G35, 70H40, 70G99, 78A35, 81E10, 81Q05, 81R25, 81R50, 81R99, 81S05, 81T15, 81T15, $81 \mathrm{~T} 75,81 \mathrm{U} 05,81 \mathrm{U} 20,83 \mathrm{~A} 05,83 \mathrm{C} 10,83 \mathrm{E} 99$

Printed on acid-free paper.

○) 2000 Springer Science+Business Media New York

Originally published by Birkhäuser Boston in 2000

Softcover reprint of the hardcover 1st edition 2000

All rights reserved. This work may not be translated or copied in whole or in part without the written permission of the publisher Springer Science+Business Media, LLC,

except for brief excerpts in connection with reviews or

scholarly analysis. Use in connection with any form of information storage and retrieval, electronic adaptation, computer software, or by similar or dissimilar methodology now known or hereafter developed is forbidden.

The use of general descriptive names, trade names, trademarks, etc., in this publication, even if the former are not especially identified, is not to be taken as a sign that such names, as understood by the Trade Marks and Merchandise Marks Act, may accordingly be used freely by anyone.

ISBN 978-1-4612-7116-1

Typeset by the editors in $\mathrm{IAT}_{\mathrm{E}} \mathrm{X}$. 


\section{Contents}

Preface to Volume 1

Rafat Abtamowicz and Bertfried Fauser ...................... vii

Preface to Volume 2

John Ryan and Wolfgang Sprößig ........................ xvii

Dedication to Gian-Carlo Rota

Bernd Schmeikal ...................................... xxiii

1 Physics - Applications and Models .................. 1

Multiparavector Subspaces of $C \ell_{n}$ : Theorems and Applications

William E. Baylis ..................................... 3

Quaternionic Spin

Tevian Dray and Corinne A. Manogue ..........................21

Pauli Terms Must Be Absent in the Dirac Equation

Kurt Just and James Thevenot ........................... 39

Electron Scattering in the Spacetime Algebra

Antony Lewis, Anthony Lasenby, and Chris Doran ................49

2 Physics - Structures $\ldots \ldots \ldots \ldots \ldots \ldots \ldots \ldots \ldots \ldots \ldots \ldots \ldots \ldots \ldots$

Twistor Approach to Relativistic Dynamics and

to the Dirac Equation - A Review

Andreas Bette ........................................ 75

Fiber with Intrinsic Action on a $1+1$ Dimensional Spacetime

Robert W. Johnson ....................................93

Dimensionally Democratic Calculus and Principles

of Polydimensional Physics

William M. Pezzaglia Jr.

A Pythagorean Metric in Relativity

Franco Israel Piazzese ................................. 125

Clifford-Valued Clifforms: A Geometric Language for Dirac Equations

Jose G. Vargas and Douglas G. Torr ........................ 135 
3 Geometry and Logic

The Principle of Duality in Clifford Algebra and Projective Geometry

Oliver Conradt

Doing Geometric Research with Clifford Algebra

Hongbo Li

Clifford Algebra of Quantum Logic

Bernd Schmeikal

4 Mathematics - Deformations

Hecke Algebra Representations in Ideals Generated by $q$-Young Clifford Idempotents

Rafat Abtamowicz and Bertfried Fauser

On $q$-Deformations of Clifford Algebras

Gaetano Fiore

Dirac Operator, Hopf Algebra of Renormalization and Structure of Space-time

Marcos Rosenbaum and J. David Vergara

Non-commutative Spaces for Graded Quantum Groups and Graded Clifford Algebras

Michaela Vancliff

5 Mathematics - Structures

Clifford Algebras and the Construction of the Basic Spinor and Semi-Spinor Modules

Johan Gijsbertus Frederik Belinfante

On the Decomposition of Clifford Algebras of Arbitrary

Bilinear Form

Bertfried Fauser and Rafat Abtamowicz

Covariant Derivatives on Minkowski Manifolds

Virginia V. Fernández, Antonio M. Moya, and

Waldyr A. Rodrigues, Jr.

An Introduction to Pseudotwistors: Spinor Solutions vs. Harmonic

Forms and Cohomology Groups

Julian Eawrynowicz and Osamu Suzuki

Ordinary Differential Equation: Symmetries and Last Multiplier

Zbigniew Oziewicz and José Ricardo R. Zeni ...

Universal Similarity Factorization Equalities Over Complex

Clifford Algebras

Yongge Tian

Index 


\section{Preface to Volume 1}

The last conference on "Clifford Algebras and Their Applications in Mathematical Physics," the 5th of this well-known series, took place in IxtapaZihuatanejo, Mexico, from June 27-July 4, 1999, in the beautiful surroundings of the Pacific Coast. The first conference of this series was organized in 1985 in Canterbury, United Kingdom, and was initiated by J.S.R. Chisholm at a time when Clifford algebras were just becoming recognized tools. Under the leadership of D. Hestenes, among others, Clifford algebras had not only entered various fields by providing an elegant and powerful tool for solving geometric problems, but, more importantly, Clifford algebras have provided a unique approach to reasoning in mathematics and physics. As a natural consequence of this development, the conferences of this series have had a large impact and have managed to form a "Clifford community."

The topics covered by the recent conference can be divided into two major parts: Clifford analysis and mathematical physics. This structure is reflected by the division of the presented contributions into two volumes: Algebra and Physics (Volume 1), and Clifford Analysis (Volume 2). The majority of papers are an outgrowth and further development of the talks given by the contributors at the conference. All papers in these two volumes have been refereed and were further developed after the conference.

There will also be a special issue of the International Journal of Theoretical Physics, edited by D. R. Finkelstein and Z. Oziewicz and containing invited papers. This too demonstrates the innovative and flourishing ideas in Clifford algebras.

During the main conference, two special sessions were organized. One was Applied Clifford Algebra in Cybernetics, Robotics, Image Processing, and Engineering (ACACSE), organized by E. J. Bayro-Corrochano and G. Sobczyk; the other was Global and Local Problems for Dirac Operators, organized by E. R. de Arellano, J. Ryan, and W. Sprößig. The goal was to gather people with interests in applications of Clifford algebras in engineering, robotics, computer vision, and symbolic computer algebra, or in the mathematics of Dirac Operators. The ACACSE activities will be presented elsewhere, while the Dirac operator contributions belong to Clifford Analysis (Volume 2).

The increasing interest in Clifford algebras has some deep foundations. Geometrical methods have seen a revival of making use of Clifford algebras 
which have been exactly designed to serve as geometrical algebras, combining the power of geometric intuition with the power of algebraic manipulations - which had been a dream of Leibniz followed by Graßmann, Peano, Hamilton, Cayley, Clifford, Boole, Rota, Hestenes, and others. It is remarkable to note the wealth of contributions to these volumes using conformal, projective, and hyperbolic geometries, not only in algebraic settings but also in Clifford analysis. The advent of these types of geometries during the 19th century gave birth to quaternions, Graßmann and Clifford algebras, and finally, to modern algebraic geometry.

Clifford algebras are used almost everywhere in mathematics and physics. Most problems can be encoded via a pair of a linear space and a quadratic form - some people even assign a value to such objects as line segments, areas, etc. However, this is already sufficient to construct a unique Clifford algebra. Multiplication makes the considered objects behave just like "numbers" and makes them easier to manipulate.

Clifford algebras have produced valuable applications. Besides their fundamental aspects, Clifford algebra, or quaternion, methods have produced well-recognized applications - even if sometimes disguised by matrix representations. Computer vision, robotics, navigation, space flight, and other areas also use these techniques.

Clifford algebras and their accompanying Graßmann-Cayley algebras broaden fields of thought. Automatic theorem proving, which might be important for autonomous robot systems, is based on this connection to Clifford algebras. Pioneered by Gian-Carlo Rota, the idea of connecting Hopf algebras with Clifford structures was also developed. Taking Clifford numbers as entities leads to new physical principles. Deformed Clifford algebras are used to solve problems in quantum field theory. Clifford algebraic computations provide a challenge for computer algebraic systems and open the new and fascinating area of experimental mathematics. Clifford algebras are bound to play a major role in quantum computing and the design of quantum computers.

Most of these currents in the Clifford community have found their way into these volumes. In this way, these books will contribute to the development of the field. The following outline will provide a subjective guide to the contributions (avoiding technical terms as much as possible) and will highlight their main features.

\section{Dedication to Gian-Carlo Rota}

Gian-Carlo Rota was invited to be a plenary speaker at this 5th International Conference on Clifford Algebras and Their Application in Mathematical Physics. His talk would have been a highlight of the conference. But he died suddenly, shortly before the conference. For this reason, the organizers of this conference arranged a special session dedicated to the memory of Professor Rota. 
Several lecturers including David Ritz Finkelstein and Bernd Schmeikal, who knew Rota personally, took a chance to present some warm remarks about Professor Rota, showing him as a person, scientist, philosopher and a beloved man.

\section{Physics - Applications and Models}

Baylis: The linear space underlying a Clifford algebra is coming conventionally equipped with multi-vector gradings. The common use is that physically different entities are mapped onto the particular gradings. The advantage of this convention is that physical transformations become inner automorphisms while the disadvantage is the introduction of possibly nonphysical or unnecessary variables. The present paper studies paravectors, which are sums of scalars and vectors and constitute themselves a graded space of a coarser grading than that of the multi-vector grading. Some mathematical outcomes are explained, and the usefulness of the paravector picture is demonstrated for plane waves and wave packets in electrodynamics. A paravector arises from a spacetime split, as discussed by Conradt.

Dray \& Manogue: Two component Weyl spinors, also extensively used by Penrose, and four component Dirac spinors are widely known in mathematics and high energy physics. Usually the latter are constructed by the former in adding two unequivalent representations of Weyl spinors, differing by the outer automorphism of complex conjugation, in a direct sum. However, the authors present a generalization of Weyl spinors by extending the base field of complex numbers $\mathbb{C}$ to the quaternions $\mathbb{H}$, a skew field and a non-commutative division ring. As an advantage, one is able to describe massless and massive Dirac equations on the same footing by two-component quaternionic spinors. As a logical step, the two-component octonionic Dirac equation, which turns out to be 10-dimensional, is examined. Dimensional reduction takes place by singling out a unit - i.e., invertible element - in the octonions, or equivalently, a unique complex subalgebra. This allows one to find the variety of particles of a generation of leptons. The three distinct possibilities for singling out a sub-quaternion algebra are conjectured to carry the three families or generations. This mechanism should be compared with Schmeikal's finding several copies of $S U(3)$ as presented in his paper.

Just \& Thevenot: As is well known, Dirac's theory contains the massterm which comes with the time-like $\gamma$-matrix. In some phenomenological models, potentials are used which are not only vectorial in nature - having a $\gamma$-matrix - but involve scalar, vector, tensor, axial or pseudo vector, and pseudo scalar contributions. Especially the anomalous magnetic moment bears a tensorial character. Such terms are called Pauli terms. This paper considers the question of whether or not such general potential is possible 
and whether it is compatible with Dirac theory. As a surprising outcome, the authors present arguments against the presence of Pauli terms. One should note, however, that these terms are not necessary in the standard model and may be rendered superfluous.

Lewis, Lasenby, \& Doran: Scattering experiments are still the common source of information in experimental high energy physics. $S$-matrix theory establishes the theoretical counterpart. An awkward and technical detail of this theory is the calculation of spin-sums of spinning particles. Using spacetime algebra (STA) - the Clifford algebra of Dirac theory - a spin direction can be introduced when the $S$-matrix is replaced by an STA operator. This operator can depend on the spin direction. In this way, a plain and straightened method is developed to work directly with the invariant spin direction avoiding spin sums and a choice of basis. However, some improvements have to be made for multi-particle spin states. Some achievements toward a proper formulation of multi-particle STA are made by the contributions of Fauser \& Abłamowicz.

\section{Physics - Structures}

Bette's contribution reviews a twistor phase space picture which was developed in the past. After having introduced the twistor phase space, the first point is the introduction of shifted position coordinates which fulfill a non-trivial Poisson bracket and are, thereby, non-commuting. These positions are physically motivated by the requirement that the inner product of momentum and Pauli-Lubanski spin-part vanish. This requirement is motivated by Dirac quantization and conservation laws. In fact, since one wants to have momenta represented by derivatives, one looks for Noether currents. A classical spinning particle is given as an example in $\mathbf{T p}(\mathbf{2})$, and the resulting equations of motion are shown to be different from BargmanMichel-Telegdi equations.

Johnson: Clifford algebras have, as an interesting sub-structure, discrete groups sometimes called Dirac groups. In fact, one can construct Clifford algebras over the reals by a ring extension from the group algebras of these cyclic and dihedral discrete groups. Based on this idea, the author tensors two such structures to get a prototype of a fiber of a configuration space manifold. After defining a suitable subspace as the tangent space, he is able to incorporate the action in the complement which finally constitutes a constraint in the tensor space. A $1+1$ dimensional model is considered.

Pezzaglia criticizes that a mere reformulation of physics in new mathematical formalisms does not lead to new physics even if there might be a great achievement in straightening out the problem at hand. However, changing the perspective might be a key step in being able to generalize physical 
principles and to reach new physics. The author's key point is to look at a Clifford element as an entity even if it can be split into multi-vectors. Therefore, he interprets every multi-vector part of a Clifford number as a physical quantity with its own coordinate. Indeed, this is the situation in quaternion theory, where the "vector" part is given by the linear span of $\mathbf{i}, \mathbf{j}, \mathbf{k}=\mathbf{i j}$, and $\mathbf{k}$ is algebraically -but not linearly- dependent. As an example, special relativity is revisited, and a "polydimensional" (ungraded) formulation of physics is developed. Multi-vector valued, or "matrix," derivatives and the action principle are formulated. Papapetrou's equation and Crawford's hypergravity provide examples of the method in classical physics.

Piazzese: A real linear space can be equipped with different metrical forms, e.g., Euclidean or Minkowski. In this contribution, a relation is established for (time-like) vectors of a Minkowski space to such a Euclidean space without resorting to complex numbers. The remarkable fact is that one is able to transport transformation laws into the Euclidean picture. This allows the author to propose a quasi-classical description of a particle. Besides the classical energy, formed with use of the relativistic velocity - i.e., quasi classical - a second term arises in this description, which can be connected - via de Broglie's idea that every particle has an "internal clock," or an internal frequency - to an internal degree of freedom. This freedom might be of rotatory nature, and it might be connected to spin. The quasi-classical energy becomes frame independent.

Vargas \& Torr provide a clear introduction to the unification program which had been developed by the authors in the past. The second section concentrates on notation not only for clarity but also to point out some difficulties, which are usually ignored, but which prove to be most important in the later development. A particular point presented in the paper is the distinction between the Dirac-Hestenes equation and Kähler theory of Dirac equations. Differential forms are introduced. Using the Hestenesian idea of mathematical viruses, two major diseases of common treatments, the "transmutation virus" and the "bachelor algebra virus" are described, and their prevention is discussed. A motivation to alter the Kähler approach by introducing a Kaluza-Klein type theory is given which is later on related to Finsler geometry. Connection is made with previous developments, pointing out a new way of deriving the results. Finally, the interior derivative in the particular case of the previously given Kaluza-Klein theory is introduced. The conclusion comes up with some very interesting outlooks about the further development of the theory. A full geometrical (invariant) Kähler equation for Clifford valued clifforms is given.

\section{Geometry and Logic}

Conradt: Duality is currently a well-recognized structure in physical theo- 
ries. In string theory, duality connects strong and weakly interacting models which allows a perturbative approach to the former. Duality originated in projective geometry where it appeared as the striking fact that every projective theorem has a dual theorem if one interchanges several notions as point with plane, join with meet, etc. Since Clifford geometric algebras are known to describe metric and projective geometries, the paper calls for an implementation of this rather fundamental projective concept. Starting by defining a Clifford algebra, the author uses Poincare duality of the underlying multi-vector space and asks the question if one could use $(n-1)$-vectors - the isomorphic picture of 1-vectors - to construct a dualized Clifford algebra. It turns out that the meet of the $(n-1)$-vectors can be seen to establish the dual outer product. In the same way, a dual Clifford product can be defined, and a dual Clifford algebra can be formulated in the same vector space as the original one, but with all $r$-multi-vectors mapped to $(n-r)$-multi-vectors of the Poincaré dual space. Such a duality for meet and join has been investigated by Rota and others. The resulting algebra is called double or Grassmann-Cayley algebra and constitutes a substructure of the Clifford approach presented here. The interpretation of such dual Clifford algebras in terms of projective geometry is given. Projective coordinate systems for points, lines, and planes are exemplified. The linear complex is introduced and a motivation provided as to where to use this structure in physics.

Li: Non-Euclidean geometry was one of the main working fields of W. K. Clifford, which might well have influenced his algebraic ideas. Furthermore, non-Euclidean geometries have already been described by Euclidean models by Felix Klein in the 19th century. This fact provides the basis of the study of hyperbolic geometries by Clifford algebraic techniques, singling out appropriate subspaces. Geometric facts can be encoded in the GrassmannCayley algebra, called "double algebra" by Rota. The main achievement of such an algebraization is that it opens an analytic and invariant approach to geometric problems. Automated geometric theorem proving is a possibility explored in this contribution. Not only machine based recalculations of theorems but the quite more interesting proving of new theorems, thus, becomes possible. Beside its beauty, this method is important also for applications in robotics and visualization.

Schmeikal: A ring of idempotent elements, or (alternatively) a ring over $\mathbb{Z}_{2}$, is a Boolean ring. Any assertions in such a ring can be interpreted as true or false, while the operations in this algebra become inference in a certain logic. Boole, de Morgan, Frege as McCulloch, Parry, or Peirce, or Zellweger have considered pictographical notations of logical conjunctions. Schmeikal adds in this contribution a description by the subring of idempotent elements of a Clifford algebra. The set of all idempotents constitutes a lattice, and, thereby, one can define "basic reflections," i.e., involutive 
automorphisms. Since representation spaces can be seen to be ideals generated by idempotents, such reflections connect different spaces. Following an idea of Chisholm, one can recover the fundamental $S U(3)$ representation by fixing one primitive idempotent out of four and then by looking at its stabilizer group. The octahedral symmetry of the idempotent lattice of the Dirac algebra is examined, and six copies of $S U(3)$ are detected. However, the picture gets more complicated by the introduction of generalized logic operators, which act on logic assertions. This tool, after being developed, is applied to establish the logic of Dirac spinors. Discrete symmetries are discussed as examples. Finally, it is outlined how this model can be enlarged to $C \ell_{n, n}$. It is pointed out that using wave functions, and not $\mathbb{Z}_{2}$, provides an example of quantum logic where "tertium datur."

\section{Mathematics - Deformations}

Abłamowicz \& Fauser: Indistinguishable particles are described by wave or partition functions invariant under the permutation group. This observation, made by physicists in thermodynamics and quantum mechanics, has influenced the theory of group representations to a large degree. Already Weyl showed that multi-particle states can be classified by a method developed by Young. Multi-particle Clifford algebras have, thus, to carry an action of the symmetric group. This article studies representations of the deformation of the group algebra of the symmetric group known as the Hecke algebra. Deformations have been proposed to serve as symmetries for composed entities. This provides the main motivation for this investigation and is in full accord with Fiore's contribution. Representations are constructed in ideals generated by $q$-idempotents in quantum Clifford algebras. These idempotents are Young operators. To get an intimate relation between both structures, reversion is taken to act as conjugation on Young operators, i.e., Young idempotents. Detailed computations are possible only by computer algebra. CLIFFORD, a Maple V package for (quantum) Clifford algebras - developed by one of the authors R.A. - has made algebraic computations possible. The representation theory in the 2and 3-dimensional cases is developed in full detail.

Fiore: Any Clifford algebra naturally has a Lie algebra substructure. Therefore, it is interesting to ask if it is possible to $q$-deform this Lie algebra, or, more precisely, its enveloping algebra $U_{q}$. As a natural outcome of covariance under the action of such a quasi-triangular Hopf algebra, one obtains a $q$-deformed Clifford algebra. However, due to finite dimensionality in the orthogonal case, it is possible to express the deformed generators as polynomials in the undeformed ones. As a consequence, $q$-deformed creation and annihilation operators might be interpreted as creation and annihilation operators of effective, or compound, entities. A detailed analysis of the situation gives a connection between deformed and undeformed invariants. 
It is shown under which conditions non-trivial deformations, having new invariants, actually occur.

Rosenbaum \& Vergara point out that recently at two different places closely related Hopf algebra structures have popped up. The first one is the Connes-Moscovici Hopf algebra, which originates from non-commutative extensions of Riemannian geometry. The second is the Hopf algebra of rooted trees, which has been employed by Connes and Kreimer to produce the combinatorics of renormalization in perturbative quantum field theory. Decorated rooted trees are used to establish the forest theorems of renormalization, and the antipode action generates the counter terms in all orders. The aim of this work is to present both types of Hopf algebras in an invariant, coordinate-free language. Furthermore, it is shown that there might be a connection between the Dirac operator, spacetime at Planck scale, and the above two Hopf algebras, which may lead toward a finite quantum field theory. The connection to Schwinger-Dyson equations is discussed.

Vancliff: Neither physics nor mathematics is currently able to present a concise model of a non-commutative space as a coordinate space of a quantum group or other deformed algebras. Common methods fail to work since in such non-commutative spaces one cannot find a Poincaré-Birkhoff-Witt like basis. Following an idea of S.P. Smith, this work, being projective and geometric in nature, connects geometric data to deformed algebras. The Sklyanin algebra serves as a model to exemplify the problem at hand. The deep connection between projective point, line, etc., schemes and quantum spaces is explored. Roughly speaking, the quantum space of an algebra is a quotient category of graded modules. Certain modules play the role of points; others play the role of lines, and so forth. In the commutative setting, this idea can be traced back to J.-P. Serre. Quantum spaces are constructed via Poisson geometry; examples are provided.

\section{Mathematics - Structures}

Belinfante: Spinors have been discovered by Élie Cartan when classifying complex semi-simple Lie algebras. During further development done by Cartan, Freudenthal, Dynkin, Chevalley, and others, it became clear that one can construct spinor modules of $\mathbf{B}$ and $\mathbf{D}$ type Lie algebras over the integers. The integers play a fundamental role in the classification also. Spinors are connected, however, to Clifford algebras constituting their natural irreducible representation spaces. As a natural approach, spinor and semi-spinor modules are constructed for complex orthogonal Lie algebras i.e., Lie algebras of types $\mathbf{B}$ and D. First and second Clifford algebras are introduced, and the mechanism using Dynkin diagrams to construct spinor modules is explicitely given. $\mathbf{B}_{1}$ and $\mathbf{B}_{2}$ provide examples; spin groups are 
discussed. MATHEMATICA code is provided which was used to check the results and invites the reader to redo the computations for gaining deeper insights into the theory.

Fauser \& Abłamowicz: Clifford algebras over real and complex numbers are classified. As an outcome of this classification, every Clifford algebra can be decomposed into graded tensor products of "atomic," i.e., indecomposable, Clifford algebra factors. These factors are at most of algebraic dimension 4. This decomposition is the origin of periodicity theorems and vice versa. Albert Crumeyrolle stated that the decomposition properties of Clifford algebras provide the Mendeleiev periodic system of elementary particles. However, the situation can be much more complicated. Defining quantum Clifford algebras, i.e., Clifford algebras of an arbitrary bilinear form, it can be shown that common periodicity theorems in general fail to hold. A detailed introduction provides arguments that this is not the exception, but, instead, it should be seen as the rule. The Wick theorem of normal-ordering in quantum mechanics and quantum field theory establishes a quantum Clifford algebra structure. After the detailed and rigorous development of the theory, three examples are provided showing the theory at work.

Fernández, Moya \& Rodrigues present in their paper the theory of covariant derivative operators on a Minkowski manifold. As a main tool of their investigation, the concept of multiform calculus is used. It is developed by passing directional covariant derivative operators and associated operators to covariant derivative operators which are compatible with a non-degenerate symmetric tensor in a Minkowski manifold. This seems to be one of the main goals of the paper because this result allows us to construct Riemann-Cartan geometries. As examples of applications of the presented theory, the Levi-Civita derivative and the Hestenes derivative are discussed in a detailed way, and they are interpreted in the framework of the developed theory. The paper presents an elegant way to handle covariant derivative operators, and it should initiate discussions about the different approaches.

Lawrynowicz \& Suzuki: Twistors, introduced by Penrose, have been successfully used in gravity, the theory of non-linear differential equations, and representation theory of conformal groups. The "twistor program" has recently been geometrized by Ławrynowicz and Rembieliński. The geometric approach makes it possible to connect the Hurwitz problem of (de)composing quadratic forms with the so-called Hurwitz pairs, which constitute pseudo twistors. These pseudo twistors can be constructed for arbitrary signature and are not necessarily connected to conformal symmetry. This contribution deals with a special class of Hurwitz twistors, which includes signature $(3,2)$ and its dual $(1,4)$. Cohomological aspects and a 
generalization of Cartan's triality to a doubled triality as an atomization theorem and holomorphic embeddings are presented.

Oziewicz \& Zeni: Symmetries can be used to reduce the order of differential equations via Lie's theorem. Sometimes this is not directly possible, but only after having prolonged the differential equation by a suitable integrating factor. The well-known multiplication of Newton's equations of motions by an $\dot{x}$ can be integrated yielding the energy conservation law. Lie had already shown under which conditions a "last multiplier" can be found, turning a differential equation into an exact one which can then be integrated. This article tackles the problem of finding last multiplier and the corresponding symmetries for ordinary differential equations in $n+1$ dimensions. Differential forms are shown to be the natural language for the problem at hand, and a generalized Lie theorem is proved. Moreover, this method is constructive and allows one to find the integrating multiplier by direct calculations. The method is independent of a Riemannian or symplectic structure and does not rely on coordinate methods.

Tian: Besides their invariant coordinate-free character, Clifford algebras are still used by many physicists via matrix representations. Starting from a generator and relations approach to complex Clifford algebras, it is shown that there are only two distinct types of representations. One is for simple Clifford algebras, if the number of generators is even, and one is over a double field, in the semi-simple case when the dimension is odd. Complex similarity factorizations provide a one-to-one mapping of the abstract algebras into certain matrix algebras. In this way, each element $a$ in the $\mathbb{C}$ algebra $C \ell_{n}$ could be regarded as an eigenvalue of its complex representation matrix $\phi_{n \times n}(a)$. This might be useful in application as calculating exponentials of Clifford numbers.

Acknowledgment: Since editing such a book cannot be done without valuable help of other people, we would like to express our gratitude to all contributors: Ann Kostant, Tom Grasso, and Caroline Graf of Birkhäuser, Elizabeth Loew of $\mathrm{T}_{\mathrm{E} X n i q u e s}$ for her help with $\mathrm{T}_{\mathrm{E} X i n g}$, Amy Knox for her monumental proofreading, and all referees for their constructive criticism.

The first editor, R.A., thanks his wife Halina for her patience during this project. The second editor, B.F., would like to thank his wife Mechthild for her support during the laborious period when the book was completed.

Rafał Abłamowicz, Cookeville, Tennessee, U.S.A.

Bertfried Fauser, Konstanz, Germany

April 1, 2000 


\section{Preface to Volume 2}

This volume of contributed papers arises from the section on Clifford analysis which took place as part of the "5th International Conference on Clifford Algebras and Their Applications in Mathematical Physics," held in Ixtapa, Mexico, June, 1999. Like in Volume 1, the majority of papers are an outgrowth and further development of the talks presented at the conference. All papers in this volume were refereed and were further developed after the conference. The editors are grateful to the referees for their extremely valuable assistance in creating this volume. The papers gathered here reflect some of the latest developments in the field of Clifford analysis and its applications. Topics range from the study of generalized Schwarzian derivatives to applications to boundary value problems and singular integrals. Topics covered also include links to a Möbius invariant function theory on hyperbolic space, analogues of Ahlfors-Beurling inequalities and their applications, differentiability properties of monogenic functions, links to supersymmetry, hyperbolic Dirac equations, and scattering theory. The papers appearing here can be broadly subdivided into the following categories.

\section{Partial Differential Equations and Boundary Value Problems}

As is well known, the complex Beltrami equation is of enormous importance in the general theory of elliptic equations and has many applications to other fields of analysis and geometry. Because of the many degrees of freedom in the combination of partial differential operators in higher dimensions, there is a large variety of generalized Beltrami equations. To motivate the treatment by Clifford analytic methods, U. Kähler shows some typical difficulties (integrability conditions, conditions on the Jacobian, etc.) in higher dimensions. The paper is a comprehensive survey of the study of Beltrami type equations in the three-dimensional setting. The results are based on integral operator methods and include detailed norm estimates for integral operators in different function spaces.

In her article, Xinhua Ji shows the relationship between Green's function, Möbius transformations and the Laplace-Beltrami operator acting on the one point compactification of $\mathbb{R}^{n}$. The author gives a complete description of all non-Euclidean translations of the corresponding Möbius group. With 
the aid of a geodesic distance, she obtains the fundamental solution of the degenerate Laplace-Beltrami equation. Finally, she solves the Dirichlet problem for the non-homogeneous Laplace-Beltrami equation. This paper contains a very nice example of a generalized Poisson kernel illustrating that the maximum principle is no longer valid for degenerate elliptic equations.

W. Sprößig considers a stationary problem from fluid mechanics using methods from Clifford analysis. Stationary Navier-Stokes equations are combined with field induction. Under certain conditions the solution can be obtained by an iteration procedure which converges rapidly in Sobolev spaces.

\section{Singular Integral Operators}

In their paper, Tao Qian, John Ryan and Xinhua Ji study Fourier multipliers and singular integral operators on Möbius images of Lipschitz graphs and starlike Lipschitz surfaces. All basic notation is explained. It is shown that the singular integral operators form an operator algebra. The main ideas are the application of a generalized Fourier transform and the use of Möbius transformations to pullback known results. One of the main results is the fact that, in the case of Lipschitz graphs, the operator algebra of singular integrals may be identified with the bounded holomorphic Fourier multipliers. The same problems are considered for starlike Lipschitz surfaces.

J. B. Reyes and R. A. Blaya study quaternionic Cauchy integrals on Ahlfors regular surfaces. In this very general setting Plemelj-Sokhotzkij formulae are deduced. These results can be applied to prove the solvability of a special case of Riemann's problem.

In M. Martin's article he proves some Hedberg type inequalities for the convolution operator associated with the Cauchy kernel in Euclidean space. Each of these inequalities involves a specific maximal operator and they all provide the best possible constants. Applications of Clifford analysis are also presented. Among them, one should single out a higher-dimensional generalization of a classical inequality in one-variable complex function theory due to Ahlfors and Beurling and some extensions of Alexander's inequality.

\section{Applications in Geometry and Physics}

S. Bernstein describes a new application for the Borel-Pompeiu formula in $\mathbb{C}^{n}$. It is obtained as a direct analogue of the Martinelli-Bochner formula. An extremely interesting feature here is her application of these results to scattering theory.

In G. Kaiser's contribution complex distance is applied to describe a useful extension of potential theory in $\mathbb{R}^{n}$ to $\mathbb{C}^{n}$. The resulting Newtonian 
potential is generated by an extended source distribution $\tilde{\delta}(z)$ in $\mathbb{C}^{n}$ whose restriction to $\mathbb{R}^{n}$ is the point source $\delta(x)$. This provides a possible model for extended particles in physics. In $\mathbb{C}^{n+1}$, interpreted as complex spacetime, $\tilde{\delta}$ acts as a propagator generating solutions of the wave equation from their initial values. This gives a new connection between elliptic and hyperbolic equations that does not assume analyticity of the Cauchy data. Generalized to Clifford analysis, it induces a similar connection between solutions of elliptic and hyperbolic Dirac equations, thereby extending earlier work of J. Ryan. There is a natural application to the time-dependent, inhomogeneous Dirac and Maxwell equations and the so-called electromagnetic wavelets.

The paper by J. Snygg deals with the use of Clifford algebras in differential geometry, especially involving the holonomy group. The Clifford algebra is used to express isometry operators.

In his paper F. Sommen presents an extension of Clifford analysis using both commuting as well as anti-commuting variables, thus, following the lines of thinking of supersymmetry. For abstract vector variables, the calculus remains the same. He illustrates that the radial algebra can be represented by both the use of commuting and anti-commuting variables. Another important fact is that the action of both the symplectic group and the rotation group are united in the super spin-group. This contribution suggests that the radial algebra is a natural background for supersymmetry because it is independent of dimension and invariant in the group theoretic sense.

J. Tolksdorf investigates bosonic and fermionic action in a Euclidean version of the standard model of particle physics in terms of elliptic Dirac operators on compact smooth even-dimensional manifolds without boundary. He explains the specific role of "standard Dirac operators" for fermionic action in contrast to the role of non-standard operators of Dirac type for bosonic action and he relates the Wodzicki residue (WR) to this action, thereby illuminating also an interesting interrelation with Connes' noncommutative geometry.

\section{Möbius Transformations and Monogenic Functions}

In their contribution M. Wada and O. Kobayashi define Schwarzian derivatives of transformations of $\mathbb{R}^{n}$ in terms of Schwarzian derivatives of regular curves and prove that a transformation of $\mathbb{R}^{n}$ is Möbius if and only if its Schwarzian derivatives are constantly zero. The framework then is modified to prove a result on immersions between Riemannian manifolds. The paper gives a new definition for Schwarzian derivative (cf. [1] and [3]) and shows accordingly the necessary and sufficient condition for a transformation being Möbius in terms of its Schwarzian derivatives.

In the last decades the construction of infinitesimal generators of the 
conformal group has played an important role in the study of monogenic functions. Several authors, including P. Lounesto, J. Ryan, F. Sommen and H. Leutwiler, have results on this topic. For instance F. Sommen [4] has obtained in his paper on monogenic operators a Taylor series formula using the conformal group. Here Y. Krasnov presents an explicit Taylor series formula by making use of the conformal group and its generators.

The paper of $\mathrm{T}$. Hempfling is devoted to the hyperbolic modification of Clifford analysis. He considers the Cauchy-Riemann operator in $\mathbb{R}^{n+1}$. By splitting this operator into a radial and spherical part, he can formulate conditions for the radial part to vanish. This is especially true if the corresponding functions are hypermonogenic.

In 1978, A. Sudbery (cf. [5]) was the first to define in a quaternionic setting the derivative as differential coefficient between two forms of higher degree. Here $\mathrm{H}$. Malonek succeeds in getting a similar result within the Clifford algebra $C \ell_{0, n}$ by considering differential forms of degree $n-1$ and $n$ as well as using the Hodge star operator; see also earlier results of J. Ryan cited in H. Malonek's paper. Here H. Malonek introduces the notion of the hypercomplex derivability of a function defined for paravectors with values in the $n$-dimensional real Clifford algebra. For instance, a real valued function $f$ is called (left) derivable if and only if $A_{f, \ell}(z) \in C \ell_{0, n}$ exists and

$$
d(d \tau f)=d \sigma A_{f, \ell}(z)
$$

where $d \sigma$ denotes a hypercomplex differential of degree $n$ and $d \tau$ a hypercomplex differential of degree $n-1$. The main result is contained in a theorem that shows that a real differentiable function $f$ is (left) derivable if and only if $f$ is (left) monogenic.

H. Leutwiler shows in [2] and some other papers that the power functions are solutions of the modified Cauchy-Riemann system

$$
x_{n} D f+(n-1) f_{n}=0 .
$$

This equation is closely related to the hyperbolic metric. Consequently, these functions are closely connected to the Laplace-Beltrami equation. H. Leutwiler and Sirkka-Liisa Eriksson-Bique introduce in their paper hypermonogenic functions as a generalization of classical complex functions. These hypermonogenic functions behave in relation to the Laplace-Beltrami operator like monogenic functions to the Laplacian. They introduce operators $P$ and $Q$ which can be understood as projections onto the real and "imaginary" parts of the Clifford algebra $C \ell_{n-1}$. The main theorems describe hypermonogenic functions, their relation to solutions of the hyperbolic Dirac operator and a representation formula.

Another paper devoted to modified Clifford analysis is presented by P. Cerejeiras. On the basis of results obtained by H. Leutwiler, J. Cnops, 
and J. Ryan, the existence of a Poisson-Szegö kernel for the LaplaceBeltrami equation associated with an $n$-dimensional hyperbolic space is proven. Poisson-Szegö kernels are explicitely constructed for a large class of orientable manifolds by using the initial kernel for the spherical model of hyperbolic spaces and the usual Möbius transform. These kernels solve a generalized Dirichlet problem.

The editors express their gratitude to Amy Knox for her very thorough proofreading of all papers.

John Ryan, Fayetteville, Arkansas, U.S.A.

Wolfgang Sprößig, Freiberg, Germany

April 1, 2000

\section{REFERENCES}

[1] L. V. Ahlfors, Cross-ratios and Schwarzian derivatives in $\mathbb{R}^{n}$, Complex Analysis, J. Hersch and A. Huber, eds., articles dedicated to Albert Pfluger on the occasion of his 80th birthday, Birkhäuser, 1988, 1-15.

[2] H. Leutwiler, Rudiments of a function theory in $\mathbb{R}^{3}$, Forum Math. 7 (1995), 279-305.

[3] J. Ryan, Generalized Schwarzian derivatives for generalized fractional linear transformations, Ann Polon. Math. LVII (1992), 29-44.

[4] F. Sommen, N. Van Acker, Monogenic differential operators, Results in Math. Vol. 22 (1992), 781-798.

[5] A. Sudbery, Quaternionic analysis, Math. Proc. Cambr. Phil. Soc. 85 (1979), 199-225. 


\section{Dedication to Gian-Carlo Rota}

Shortened version of a presentation given at the Special Session dedicated to the memory of Gian-Carlo Rota at the 5th International Conference on Clifford Algebras and their Applications in Mathematical Physics, Ixtapa, Mexico, June 27 - July 4, 1999.

Gian-Carlo Rota - philosopher and mathematician - inspired and befriended several generations of colleagues and students; indeed his presence continues to impact on many of us even after his death.

Rota the man and Rota the highly original thinker drew from his foundations in phenomenological thinking, namely, the traditions of Husserl and Heidegger whose works became Rota's "livres de chevet" throughout his life. From phenomenological traditions, Rota developed an altogether unique philosophy of mathematics, which is engagingly presented in the profound essays from his book Indiscrete Thoughts. Indiscrete Thoughts is also an autobiographical accounting of Rota's tastes, what and whom he admired or disliked, his perspectives on the golden age of 20th century science, and his intense friendship with Stan Ulam (intricately exposed in The Lost Café).

Ulam's death created a deep void in Rota. Although he had always realized that death is a natural part of life, the sadness that would accompany such a realization left him with a sense of "amertume." The art of dying as the art of living was important, and in Ulam's passing, Rota sought to extend Stan's influence beyond the grave. As he said, "I am at a loss to tell where Ulam ends and where I really begin. Perhaps this is one way he chose to survive." It is a rather phenomenological statement, taken more from a living experience than from science, and akin perhaps to the notion of existential psychology and interpersonal perception - concepts dear to Rota.

In connection with interpersonal perception, Rota describes how Ulam used to comfort him when he was feeling depressed about death. Ulam would say to Rota, "You are not the best mathematician I ever met because von Neumann was better. You are not the best Italian I have ever met because Fermi was better. But you are the best psychologist I have ever met," [1] in an attempt to lighten his mood. This devotion helped ease the transition from life to death. 
Ulam also influenced Rota's mathematical ideas to some extent through his phenomological "flashes of foresight." [2] One such idea involved the concept of surface. In the nineteenth century, mathematicians conceived of a surface by explicit equations. After some further abstraction, topologists redefined the concept in terms of extension and neighborhood. Rota and N. Metropolis [3] picked up an essential part of the problem, namely codification and enumeration of the faces of the $n$-cube. Their paper $O n$ the lattice of the faces of the $n$-cube makes us aware of the Dilworth partition and its symmetries.

Along with Ulam, Rota further envisioned a refinement of Maxwell's equations that would rid them of algebraic irrelevancies. Such equations can be found in Clifford Algebras and Spinors [4], by Pertti Lounesto. Rota believed there were differences in algebra one, consisting of algebraic geometry or algebraic number theory, and algebra two, connected with Boole's name and containing logic algebra and invariant theory. Recent developments in Clifford algebra have removed those differences that once seemed invincible. Rota himself made advances in algebra two.

With Rota's departure we are left alone to decipher the symbols of instant vision and their relations to phenomenology and what is meant by them. Following Husserl, Rota proposed to investigate connectives of the form "A is absent from B," or "A anticipates B." The meaning of "anticipation" apparently bridges living experience and mathematics.

The culmination of Rota's ideas on death, life, and God came in April 1999 with his own death. It seems that Rota had at last managed to connect the big with the little death, sleep with eternity. We live with fond memories of Gian-Carlo: his manifold generosities and kindnesses, his lifelong quest for understanding and truth, and his perceptions of mathematics and its relation to logical and clear thinking.

Note. This dedication was shortened and reformulated in part by Ann Kostant, Executive Editor of Mathematics and Physics, Birkhäuser.

\section{REFERENCES}

[1] Gian-Carlo Rota, Indiscrete Thoughts, Birkhäuser, Boston, 1997, ch. 6: The Lost Café, p. 83.

[2] Ronald D. Laing, Conversations with Children, Harmondsworth, 1978, p. 19. 
[3] N. Metropolis, Gian-Carlo Rota, On the lattice of faces of the $n$-cube, Bulletin of the American Mathematical Society Vol. 84, No. 2. March 1978.

[4] P. Lounesto, Clifford Algebras and Spinors, Cambridge University Press, Cambridge, 1997.

Bernd Schmeikal

Biofield Laboratory

Kundmanngasse 26, A-1030 Vienna, Austria

Email: schmeika@isis.wu-wien.ac.at

Received October 4, 1999; Revised: April 28, 2000 